\title{
The Role of Surgery in Epithelial Ovarian Cancer
}

\author{
Spiliotis J MD, PhD, FASPSM ${ }^{1,2 *}$ \\ ${ }^{1}$ Department of Surgical Oncology and HIPEC, Athens Medical Centre, Athens, Greece \\ ${ }^{2}$ Department of Surgical Oncology and HIPEC, European Interbalkan Medical Centre, Thessaloniki, Greece
}

${ }^{\star}$ Corresponding author: Spiliotis J, Department of Surgical Oncology and HIPEC, Athens Medical Centre, Athens, Greece; Tel: +306942404014; E-mail: jspil@hotmail.gr

Received: February 08, 2021; Accepted: February 14, 2022; Published: February 21, 2022

\begin{abstract}
The standard of care advanced ovarian cancer is complete surgical cytoredution followed by systemic chemotherapy. Most important factor is the correct pre-surgical staging in order to choice the optimal therapeutic route. Complete tumor cytoreduction has shown an improvement in survival. Optimism patient selection for primary cytoreduction, the role of neo-adjuvant chemotherapy with interval cytoreduction and the role of secondary cytoreduction in relapse disease are the main topics of this article.
\end{abstract}

Keywords: Ovarian cancer, Cytoreductive surgery, Neoadjuvant chemotherapy

\section{Introduction}

Ovarian cancer remains a lethal cancer among the women and is the $7^{\text {th }}$ most common cancer and the $8^{\text {th }}$ cause of death worldwide [1]. Every year 300.000 new cases and 158.000 deaths are observed all around the world. Despite of multitude of genomic and medical advances in the understanding and management of E.O.C. over the past 20 years, the primary cytoreductive surgery remains one of the most important prognostic factors for overall survival [2]. Ovarian cancer arises from ovarian surface and spreads by exfoliation or through the abdominal or pelvic lymphatic system, finally cancer cells initially implant throughout the pelvis, right parabolic gutter and across the right diaphragm to the great omentum and gastrointestinal organs $[3,4]$.

Therefore complete resection of all visible disease has become the goal standard of surgery [5]. There are 3 types of surgery. Primary cytoreduction, when the disease is removed upfront before any treatment, interval cytoreduction after 3 or 4 cycles of neo-adjuvant chemotherapy and secondary cytoreduction for the cases of cancer rearrange.

\section{Primary Cytoreduction}

The treatment of a new diagnosis of ovarian cancer is a complete cytoreductive surgery and platinum/taxane systemic chemotherapy followed by maintenance therapy with PARP inhibitors or bevacizumab.

Cytoreduction may include a variety of surgical procedures, as peritonectonies, as described by Sugarbaker in middle 90's [6]. Some times in $30-50 \%$ of cases recto sigmoid resections is necessary [7]. The completeness of cytoreduction in most important factor which improves the overall survival and depends on the location of tumor spread (upper abdomen), surgical team experience and patients performance status and comorbidities [8]. Complete Ro (CCo) cytoreduction offers a longest median overall survival 64 months versus 29 months in women with less of $1 \mathrm{~cm}$ residual disease. This aggressive surgery is associated with increased morbidity but not increase mortality [9]. Laparoscopy is a useful tool to predict cytoreduction feasibility and outcomes. The evaluation of peritoneal cancer index (PCI) score to evaluate the achievement of Completeness of Cytoreduction zero (CCo) is the main procedure in our institution. Patient with E.O.C. and laparoscopic PCI less than 12 is the main factor to perform primary cytoreduction [10].

Fagotti et al. [11,12] proposed a Predictive Index Value (PIV) based on objective parameters determined during the precytoreduction laparoscopy. With this modal, the likelihood that a patient would have a suboptimal surgical result (PPV) is 100\% with a PIV $\geq 8$. They evaluated several features and gave to them a score: peritoneal carcinomatosis (score 0 for carcinomatosis involving a limited area and surgically removable by peritonectomy; score 2 for unrespectable massive peritoneal involvement and with a military pattern of distribution), diaphragmatic disease (score 0 for no infiltrating carcinomatosis and no nodules confluent with the most part of the diaphragmatic surface;) score 2 for widespread infiltrating carcinomatosis and no nodules confluent with the most part of the diaphragmatic surface), mesenteric disease (score 0 for no large infiltrating nodules and no involvement of the root of the mesentery as would be indicated by limited movement of the various intestinal segments; score 2 for large infiltrating nodules or involvement of the root of the mesentery indicated by limited movement of the various intestinal segments), omental disease (score 0 for no tumour diffusion observed along the omentum up to the large stomach curvature; score 2 for tumour diffusion observed along the omentum up to the large stomach curvature), bowel infiltration (score 0 for no bowel resection was assumed and no military carcinomatosis on the ansae observed; score 2 for bowel resection assumed or military carcinomatosis on the ansae observed), stomach infiltration (score 0 for no obvious 
neoplastic involvement of the gastric wall; score 2 for obvious neoplastic involvement of the gastric wall) and liver metastases (score 0 for no surface lesions; score 2 for any surface lesion).

Two main anatomical areas are important during primary cytoreduction, the mesenteric roof and disease above the diaphragm. In our institution for all mesenteric areas we destroy the implants with ablation using argon beam coagulator as useful adjunct to traditional surgery. The diaphragmatic stripping is the main procedure used in upper abdominal cytoreductive surgery with a 10$15 \%$ of diaphragmatic partial resection [13]. In conclusion primary cytoreduction can be done in the primary setting of treatment for advanced ovarian cancer in an experience surgical unit.

\section{Interval Cytoreduction}

In many patients some factors makes primary cytoreduction difficult to achieve complete (CCo) resection. These patients are candidates for neoadjuvant chemotherapy. The role of neoadjuvant chemotherapy is to improve the perioperative morbidity and down staging the tumor to achieve optimal results. A potential problem using chemotherapy before surgery is the formation of fibrosis and liver chemo sensitivity (chemo-liver) which will make the operation more difficult [14].

Thera are two randomized, controlled, prospective trials conducted by the (EORTC) and The Medical Research Council (MRC) Clinical trials Unit, which show no significant differences in overall survival between the groups with primary cytoreductive surgery and the one with neoadjuvant chemotherapy before surgery. Vergote et al. (14), showed no differences in mortality between the groups that underwent incomplete primary cytoreduction and the one that received neoadjuvant treatment before surgery. The median overall survival (OS) was 29 months and 30 months, respectively. The median progression free survival (PFS) was 12 months for both groups. The overall survival was higher in the group who achieved complete primary surgery. The most frequent sites for residual disease after primary or interval surgery are the diaphragm, the abdominal peritoneum and the pelvis (pouch of Douglas, uterus, bladder, rectum, and sigmoid).

The laparoscopy-based score of Fagotti et al. [15] has an important role in the prediction of optimal cytoreduction among women undergoing interval cytoreductive surgery. With a PIV $>4$, the probability of optimally resecting the disease at laparotomy was equal to 0 . Within the rate of 3-6 cycles, each incremental chemotherapy cycle was associated with a decrease in 40.1 months in median survival, so the surgery ought to be done as early in the treatment programme as possible [16]. Some guides recommend three cycles of chemotherapy. After this, patients undergo surgery, and receive another three cycles after it [17]. In conclusion the performance status, co-morbidities and surgeon experience are the main factors to decision for apply neoadjuvant chemotherapy.

\section{Secondary Cytoreduction}

Almost more than $50 \%$ of patients with EOC will have a recurrence. Recurrent ovarian cancer is treatable but rarely curable. (RR) The recurrent rates depend on the stage at initial diagnosis reaching $10 \%$ in stage I, 30\% in stage II, $70-90 \%$ for stage III and $90-95 \%$ for stage IV (10). The main factor of recurrence is the tumor biology, the chemo sensitivity and the completeness of primary/interval cytoreduction. Recently our group demonstrates that a statistical significant difference in survival relapse ovarian cancer between RESIDUAL (incomplete cytoreduction) disease and RECURRENT (after CCo reaction) disease (9). There are four types of patients depending on the recurrence (16): the ones who progress during the chemotherapy treatment, called platinum-refractory patients; the ones who progress in the first 6 months after the drug treatment, called platinumresistant patients [18]. The ROVAR score [19] includes four variable and is designed for predicting recurrence after primary treatment with surgical cytoreduction and platinum-based chemotherapy. These four variables are tumour stage at diagnosis, tumour grade at diagnosis, CA 125 serum levels at diagnosis and the presence of residual disease on CT scan after chemotherapy treatment. The ROVAR score has a sensibility and specificity of $94 \%$ and $61 \%$, respectively. It is suggested by some researchers as it is not still validated. The most important theoretical result concerning the benefit of secondary cytoreduction is more likely to be effective and removal of poor vascularized disease, eliminating pharmacological sanctuarities. A study by Van de Laar et al. [20], in which two predictive models of complete secondary cytoreductive surgery were evaluated, showed that a good performance status and the absence of ascites were two prognostic factors associated with complete secondary surgery. They conclude that more studies are needed before these two predictive models can be applied in daily clinical practice. This study also showed the importance of complete secondary cytoreduction surgery, with a better survival rate in patients with complete resection than in patients who underwent incomplete secondary cytoreductive surgery.

Chi et al. [21] give guidelines and selection criteria to select patients for secondary cytoreduction in recurrent, platinum-sensitive EOC. The goal is to achieve less than $0.5 \mathrm{~cm}$ residual disease. For operable patients, the selection criteria suggested are as follows: for patients with only one site of recurrence, with a disease-free interval of 6 months, secondary cytoreduction is the best option; from patients with multiple recurrence sites but no carcinomatosis with a diseasefree interval of 12 months, secondary cytoreduction must be offered; and, for patients with carcinomatosis who have a disease-free interval of at least 30 months secondary cytoreduction is also beneficial. They do not recommend offering secondary cytoreduction to patients who have a disease-free interval from 6 to 12 months with carcinomatosis. For patients who have multiple sites of recurrence and a diseasefree interval from 6 to 12 months or who have carcinomatoiss with a disease-free interval of 13 to 30 months, secondary cytoreduction may be considered, and the decision may be individualized based on various factors, such as the exact disease-free interval (closer to 6 or to 30 months), patients age, performance status, overall general medical condition, and the patient's preferences.

Response rate to second line chemotherapy after recurrence for platinum-sensitive patients is $30 \%$ or more, while for platinumresistant patients, the response rate is lower (from 10 to 25\%) [22]. Braicu et al. [23] compared primary with secondary cytoreduction. 
Complete tumour debulking was achieved more often during primary surgery ( $77 \%$ vs. $50 \%)$ with equivalent morbidity, but with maximal surgical effort, residual effort, residual tumour significantly correlates between the two procedures. Residual tumour after primary surgery was related with residual tomour after secondary cytoreduction. Patients with recurrence have significantly higher rates of involvement of the gastric serosa, serosa of small bowel, and mesentery. As shown, there are heterogeneous opinions and results of different studies. There are two multicentric and international studies, GOG 213 (a phase-III randomized controlled trial of carboplatin and paclitaxel alone or in combination with bevacizumab followed by bevacizumab and secondary cytoreduction surgery in platinum-sensitive recurrent ovarian, peritoneal primary and fallopian tube cancer) and DESKOP III AGO-OVAR (a randomized trial evaluating cytoreductive surgery in patients with platinum-sensitive recurrent ovarian cancer) that will define the results and indications in this heterogeneous group of patients.

\section{Future Directions}

There are five main types of ovarian carcinoma based on molecular genetic alterations that account for the 95\% of the cases: high-grade serous (70\%), endometrioid (10\%), clear cell (10\%), mucinous (3\%), and low-grade serous carcinomas $(<5 \%)$ [24]. They all have different epidemiology, genetic risk factors, precursor lesions, patterns of spread, molecular events during oncogenesis, response to chemotherapy, and prognosis. The different cellular mechanisms associated with ovarian oncogenesis and progression are that target of this new therapies [24]. There are other new molecular therapies being developed: antifolate receptor-mediated therapies (ferletuzumab, EC 145), death receptor-mediated (conatumumab) and histone and histone deacetylase (HDAC) inhibitors (vorinostat, valproic acid). In addition, antibody-based tumour vaccines and cytokine-based therapies have verified an improvement in host immune activity in order to eradicate cancer cells $[25,26]$.

\section{References}

1. Ushijima K (2010) Treatment for recurrent ovarian cancer at first relapse. Journal of Onology. [crossref]

2. Straubhar A, Chi D, Long KR (2020) Update on the role of surgery in the management of advanced epithelial ovarian cancer. Clinical Advances in hematology \& Oncology 18: 11. [crossref]

3. Romanidis K, Nagorni EA, Halkia E (2014) The role of cytoreductive surgery in advanced ovarian cancer: the general surgeon's perspectiva. J BUON 19: 598-604. [crossref]

4. Martin-Camean M, Delgado-Sanchez E, Pinera A (2016) The role of surgery in advanced epithelial ovarian cancer.

5. Narasimhulu DM, Khoury-Collado F, Chi DS (2015) Radical surgery in ovarian cancer. Curr Oncol Rep 17: 16 [crossref]

6. Sugarbaker P (1995) Peritonectomy procedures. Ann Surg 22: 29-42. [crossref]

7. Perlatka P, Sienko J, Czajkowskik (2016) Results of optimal debunking surgery with bowel resection in patients with advanced ovarian care. Worldwide J Surg Oncol 14: 58.

8. Laios A, Gryparis A, Leach C (2020) Predicting complete cytoreduction for advanced ovarian cancer patients using nearest-neighbor models. J Ovarian Res 13: 117 [crossref]

9. Spiliotis J, Iavazzo Ch, Kopanakis D, Christopoulou A (2019) Secondary debulking for ovarian carcinoma relapse: The R-R dilemma-is the prognosis different for residual or recurrent disease. [crossref]
10. Jafari MD, Halabi WJ, Stamos MJ, Nguyen VQ (2014) Surgical outcomes of hyperthermic intraperitoneal chemotherapy: Analysis of the American College of Surgeons national surgical quality improvement program. JAMA Surg 149: 170-175[crossref]

11. Fagotti A, Ferrandina G and Fanfani F (2006) A laparoscopy based score to predict surgical outcome in patients with advanced ovarian carcinoma: A Pilot Study. Ann Surg Oncol 13: 1156-1161 [crossref]

12. Fagotti A, Vizzielli G, Constantini B (2011) Learning curve and pitfalls of a laparoscopic score to describe peritoneal carcinomatosis in advanced ovarian cancer. Acta Obstet Gynecol Scand 90: 1126-1131 [crossref]

13. Ye S, He I, Liang S (2017) Diaphragmatic Surgery and related complications in primaus cytoreduction for advanced Ovarian, tubal and peritoneal carcinoma. BMC Cancer 17: 317 [crossref]

14. Vergote I, Trope CG, Amant F (2010) Neoadjuvant chemotherapy or primary surgery in stage III or IV ovarian cancer. $N$ Engl J Med 363: 943-953 [crossref]

15. Rutten MJ, Van de Vrie R, Bruining A (2015) Predicting surgical outcome in patients with international federation of gynecology and obstretics stage III or IV ovarian cancer using computed tomography. A systematic review of prediction models. Int $J$ Gunecol Cancer 25: 407-415 [crossref]

16. Bristow RE, Chi DS (2006) Platinum-based neoadjuvant chemotherapy and interval surgical cytoreduction for advanced ovarian cancer: a meta-analysis. Gynecol Oncol 103: 1070-6 [crossref]

17. Oncoguia SEGO: Cancer Epitelial de ovario, trompa y peritoneo. Guias de practica clinica en cancer ginecologico y mamario Publicaciones SEGO. Octubre 2014

18. Rutten MJ, Van de Vrie R, Bruining A (2015) Predicting surgical outcome in patients with international federation of gynecology and obstetrics stage III or IV ovarian cancer using computing tomography: a systematic review of prediction models. Int $J$ Gynecol Cancer 25: 407-415 [crossref]

19. Rizzuto I, Stavraka C, Chatterjee J (2015) Risk of ovarian cancer relapse score: a prognostic algorithm to predict relapse following treatment for advanced ovarian cancer. Int J Gynecol Cancer 25: 416-422.

20. Van de Laar R, Massuger LF, Van Gorp T (2015) External validation of two prediction models of complete secondary cytoreductive surgery in patients with recurrent epithelial ovarian cancer. Gynecol Oncol 137: 210-215 [crossref]

21. Chi DS, McCaughty K, Diaz JP (2006) Guidelines and selection criteria for secondary cytoreductive surgery in patients with recurrent, platinum-sensitive epithelial ovarian carcinoma. Cancer 106: 1933-1939

22. Vargas-Hernandez VM, Moreno-Eutimio MA, Acosta-Altamirano G (2014) Management of recurrent epithelial ovarian cancer. Gland Surg 3: 198-202 [crossref]

23. Braicu El, Sehouli J, Richter R (2012) Primary versus secondary cytoreduction for epithelial ovarian cancer: a paied analysis of tumour pattern and surgical outcome. Eur j Cancer 48: 687-694 [crossref]

24. Ziebarth AJ, Landen CN, Aalvarez RD (2012) Molecular/genetic therapies in ovarian cancer: future opportunities and challenges. Clin Obstet Gynecol 55: 156-172 [crossref]

25. Konner JA, Bell-McGuinn KM, Sabbatini P (2010) Farletuzumab, a humanized monoclonal antibody against folate receptor alpha, in epithelial ovarian cancer: a phase I study. Clin Cancer Res 16: 5288-5295 [crossref]

26. Schmeler KM, Vadhan-Raj S, Ramirez PT (2009) A phase II study of GM-Csf and rlFN-gammalb plus carboplatin for the treatment of recurrent, platinum-sensitive ovarian, fallopian tube and primary peritoneal cancer. Gynecol Oncol 113: 210-215 [crossref]

Citation:

Spiliotis J (2022) The Role of Surgery in Epithelial Ovarian Cancer. Cancer Stud Ther J Volume 7(2): 1-3. 